\title{
Studies on Malarial Sporozoites. I. Effect of Various Environmental Conditions
}

\author{
Richard J. Porter, Raymond L. Laird' and Elizabeth M. Dusseau \\ Department of Tropical Diseases, School of Public Health, Lniversity of Michigan, \\ Ann Arbor, Michigan
}

The studies reported here were initiated for primarily practical reasons. 'The principal aim was to determine procedures for induction of Plasmodium gallinaceum infections with sporozoites on a scale suitable for mass testing of anti-malarial drugs. The method of Porter (1942) as modified by Coggeshall, Porter, and I,aird (1944) had shown occasional failures in large-scale work, and it was clear that some of the manipulations involved were potentially injurious to sporozoites. Therefore, although some phenomena of general interest were observed in these studies, their main value lies in the development of reliable quantitative methods for production of malarial infections with sporozoites.

\section{Materials AND Methods}

\section{Technical Methods and Materials}

The 8-A strain of Plasmodium gallinaceum was used throughout. The mosquitoes were Aedes aegypti and A. albopictus of strains maintained in this laboratory for more than three years. The vertebrate hosts were domestic chickens, mostly White Leghorn cockerels. They were 2 to 7 days old at inoculation. In some experiments female White Leghorns were used, and occasionally chicks of other breeds, White or Barred Rocks or New Hampshire Reds. In these and many other experiments no differences in infection were observed with differences in sex or breed.

The mosquitoes were reared in the laboratory by the general method of Shannon and Putnam (1934). They were infected by feeding on chicks harboring $P$. gallinaceum of a continuous blood-passage strain subinoculated twice weekly. They were held at about $28^{\circ} \mathrm{C}$ for 10 days or more before use for infection.

Since the individual experiments concern specific aspects of technique,

1 Now in the Division of Preventive Medicine, University of Tennessee College of Medicine, Memphis, Tenn. 
no comprehensive exposition of methods is given here. The general procedure, of which individual aspects were modified in particular experiments, was as follows: Mosquitoes were immobilized with minimal exposure to anesthetic ether. The heads were cut off and a small amount of thoracic contents was squeezed out into a drop of $0.85 \%$ sodium chloride solution. The tissue thus removed is mostly fat, but the salivary glands are usually included. This bit of tissue was placed in a shell vial containing $0.5 \mathrm{ml}$ of heparinized normal chick blood $(0.85 \%$ saline $0.05 \mathrm{ml}$, powdered heparin about $20 \mathrm{mg}$, blood $0.45 \mathrm{ml}$ ). Three lengths of glass rod were placed in the vial, which was closed with a red rubber stopper and rotated on its long axis for 30 minutes at about $50 \mathrm{rpm}$. This "roller mill" distributes the sporozoites throughout the suspending medium with reasonable uniformity. Dried, giemsa-stained films of the suspension were examined and the sporozoites and erythrocytes were enumerated in a series of microscopic fields. The figure obtained ( $x$ sporozoites per 10,000 erythrocytes) was multiplied by 100,000 to give the total number of sporozoites in the suspension. This calculation makes the sufficiently accurate assumption that $0.45 \mathrm{ml}$ of normal chick blood contains about $1,000,000,000$ erythrocytes. Appropriate dilutions of the suspension were inoculated intravenously, usually in $0.2 \mathrm{ml}$ amounts. Resulting infections were compared in terms of either the prepatent period to microscopic parasitemia or the intensity of blood infection on the sixth or seventh day after inoculation.

\section{Graphical Presentation}

The data are mostly presented graphically in two ways. The first type of graph, illustrated by Fig. 1, compares the parasitemias in inoculated chicks (mean of 4 or more chicks per group) on a given day after infection. The second type of graph, exemplified by Fig. 2, compares prepatent periods in inoculated chicks (groups of 4 or more). In some cases there was 1 day's difference in prepatent periods among individual chicks in a group. In such cases, as shown in the second black bar in Fig. 2, the bar is of a height intermediate between the two prepatent periods.

Certain conventions used in the graphs require explanation. The pointed bars, as in Fig. 2, indicate that observations were discontinued on the day indicated, with the chicks still uninfected as determined by microscopic examination. The stepped bars, as in Fig. 3, show that some chicks became infected on the day indicated by the step, others remaining negative. Bars extending down from the baseline, as in Fig. 1, Ex- 
periment $I$, mean parasitemias of $0 / 10,000$ cells, not reproducible on a semilogarithmic scale. It should be noted that the graphs based on parasitemia have various baselines, selected merely for convenience in comparison of data.

A tenfold reduction of parasitemia in a graph of the type of Fig. 1 is roughly equivalent to a 1-day increase in prepatent period in a graph such as Fig. 2. It should be borne in mind, therefore, that graphs based on prepatent period show large reductions in sporozoitc infectivity by a small increase in bar height. Those based on parasitemia reveal small reductions in sporozoite infectivity by a large decrease in bar height.

\section{ResUlts \\ Methods of Immobilizing Mosquitoes}

Failure in some early mass inoculations of sporozoites suggested that the general technique outlined above was faulty. Examination of smears from unsuccessful experiments showed microscopically abnormal sporozoites. These sporozoites were either ghostlike, with pale pinkish cytoplasm and no visible nucleus, stubby or swollen, or with heavily-staining, droplet-like, rather than granular, nuclei. Similar abnormalities were seen in some of the sporozoites in smears from occasional infective suspensions, but only when more than one lot of mosquitoes had been used in the suspension. This suggested that certain lots of mosquitoes were not infective but that infection could result from a suspension containing both infective and non-infective lots. Histories of the mosquito lots showed no consistent differences between infective and non-infective ones. The possibility was apparent that manipulation of the individual lots at time of dissection, such as excessive exposure to ether, was responsible.

Figure $1^{2}$ gives results of three experiments in which randomly selected mosquitoes from the same lots were immobilized with either (a) an exposure to ether just sufficient to immobilize or (b) an exposure just sufficient to kill the mosquitoes. Other technical methods were as described above (materials and methods). Duplicate samples of mosquitocs, dissected at different times in the experiment, were used in each case to rule out differences in the infectivity of the samples themselves. It is clear that in all experiments exposure to enough ether to kill the mosqui-

2 The methods of graphical presentation are discussed in the section on Graphical Presentation of Materials and Methods. 
toes reduced the infectivity of their sporozoites as measured by the parasitemia on the sixth (Experiments I and II) or seventh (Experiment III) day after inoculation.

Figure 2 gives results of an experiment comparing several methods of immobilization. The cigarette smoke and the pyrethrum (a 3\% aerosol with sesame oil in freon) were blown into tubes containing the mosquitoes. The DDT was dissolved in carbon tetrachloride and coated

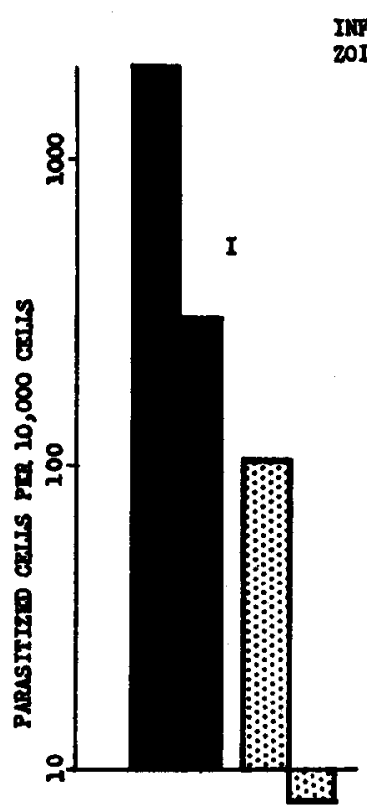

INTECTIVITY of SPOBD-

2OITES TEON BTHERTED

usquiroses
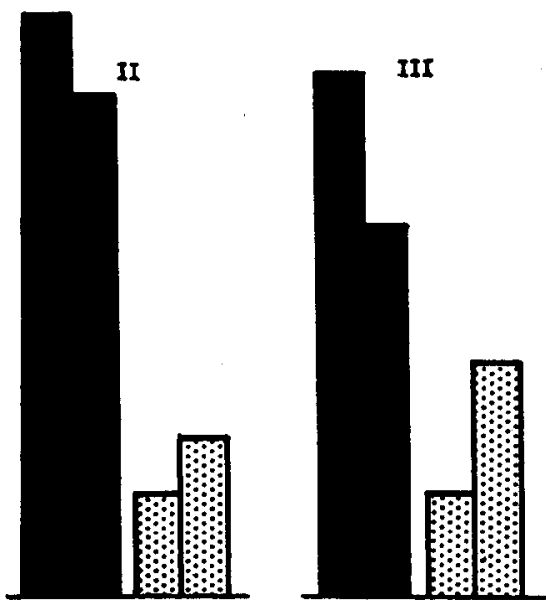

Frg. 1

heavily on the tubes before introduction of the mosquitoes. Exposure in all these cases was $2 \frac{1}{2}-3$ hours. The DDT-treated mosquitoes had to be etherized lightly, for they were still active. They were wet, and the consequent difficulty in dissection probably explains the longer prepatent period in the first sub-sample. Aside from this questionable group, it is apparent that none of the methods tested seriously reduced infectivity of the sporozoites except the one-hour etherization, which apparently destroyed all infectivity.

The results shown here with extended exposure to ether have been confirmed in two similar experiments with exposure to excess ether for 


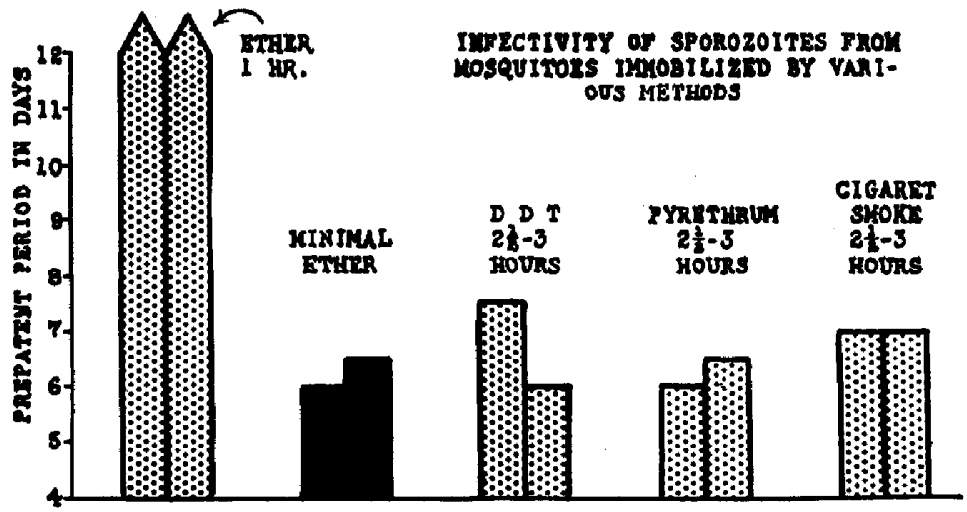

FIg. 2
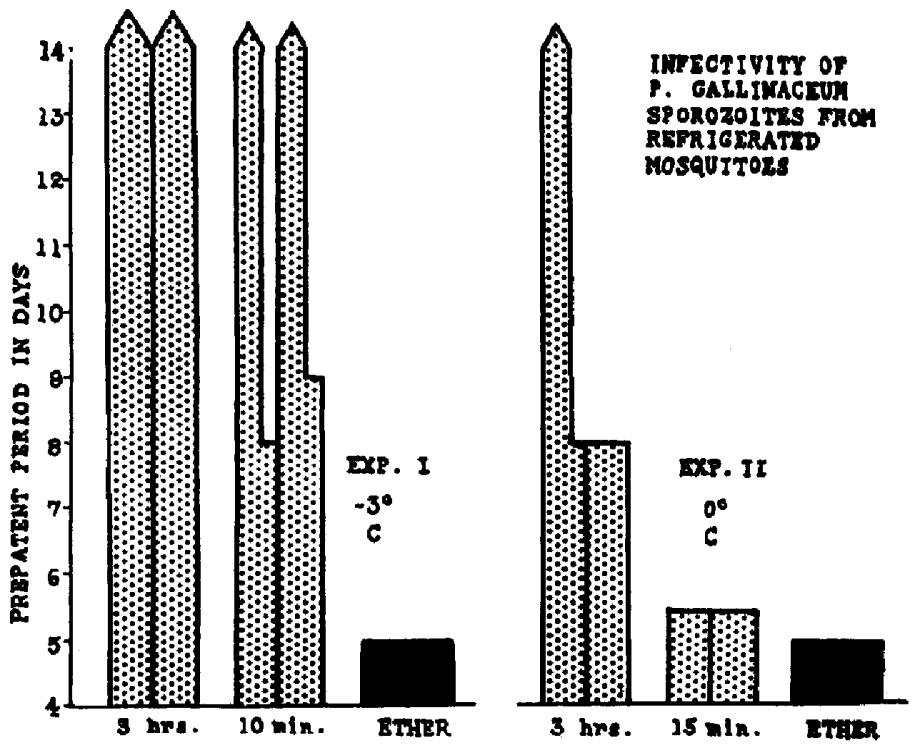

FIG. 3

30 minutes. In these cases the controls (light etherization) showed prepatent periods of 5 and 7 days respectively, while the heavily etherized lots showed no infection for at least 10 and 12 days respectively.

Figure 3 gives the results of two experiments on refrigeration at temperatures near $0^{\circ} \mathrm{C}$ as a means of immobilizing mosquitoes. It is 
clear that killing mosquotoes by chilling reduced the infectivity of their sporozoites as measured by the prepatent periods in inoculated chicks.

Additional data on the effect of low temperatures are given in Fig. 4. In all cases the chilling was for 3 hours. The mosquitoes held at $-5^{\circ} \mathrm{C}$ in this experiment, all refrigerated mosquitoes in Experiment I (Fig. 3), and these held at $0^{\circ} \mathrm{C}$ for 3 hours in Experiment II (Fig. 3) were dead at dissection. In all three experiments recorded it would appear that temperatures at or below $\mathrm{O}^{\circ} \mathrm{C}$ were lethal to sporozoites, while higher refrigerator temperatures had little or no effect.

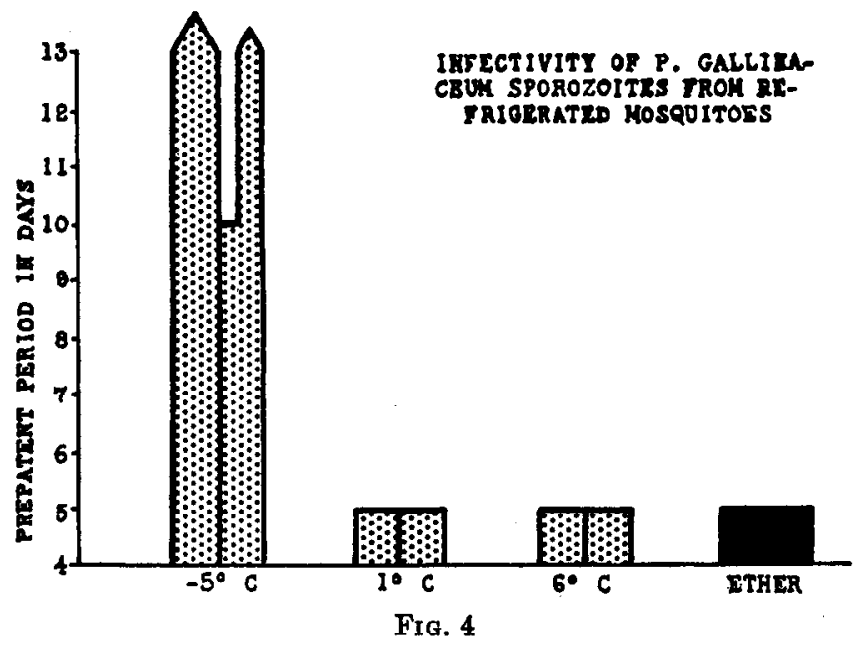

The following general conclusions may be drawn from this series of experiments. Cigarette smoke, pyrethrum aerosols, minimal etherization or minimal refrigeration at temperatures above freezing may be used for immobilization of infected mosquitoes. In excess, either refrigeration or exposure to ether reduces the infectivity of sporozoites.

Dissecting and Suspending Media for Sporozoites, and the Effects of Environmental Temperature

Figure 5 gives the results of two experiments in which salivary glands were dissected and suspended in sodium chloride solutions. Controls were handled entirely by the general procedure given in "Materials and Methods." Since both test and control groups were dissected in saline solutions, the differences being examined are those resulting from sus- 
pension in saline as compared with blood. The adverse effects of 0.21 , 0.43 , or $0.85 \%$ saline are clear.

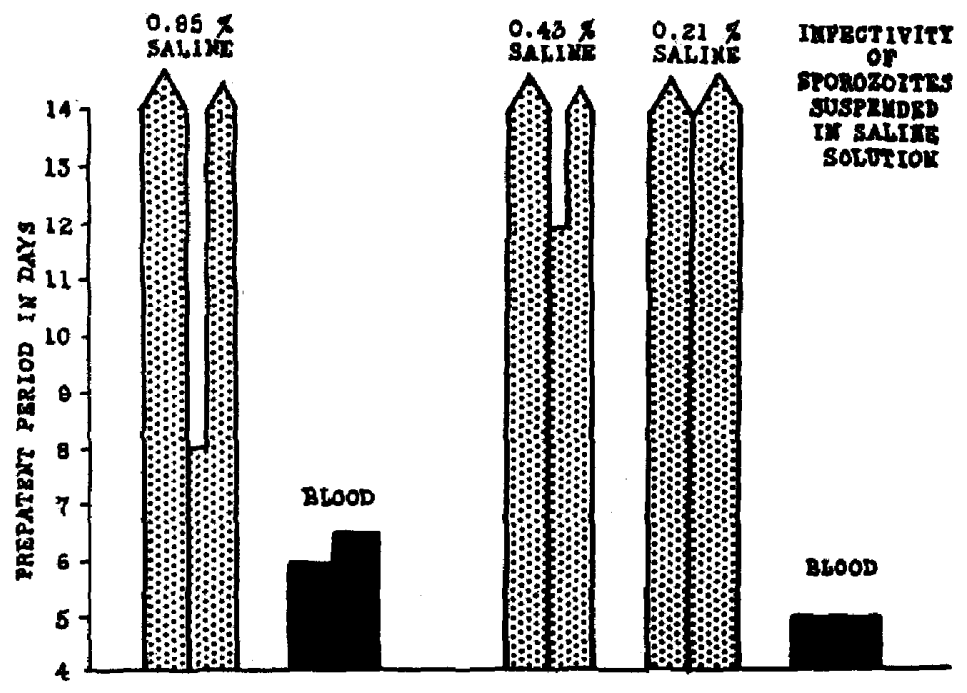

FIG. 5

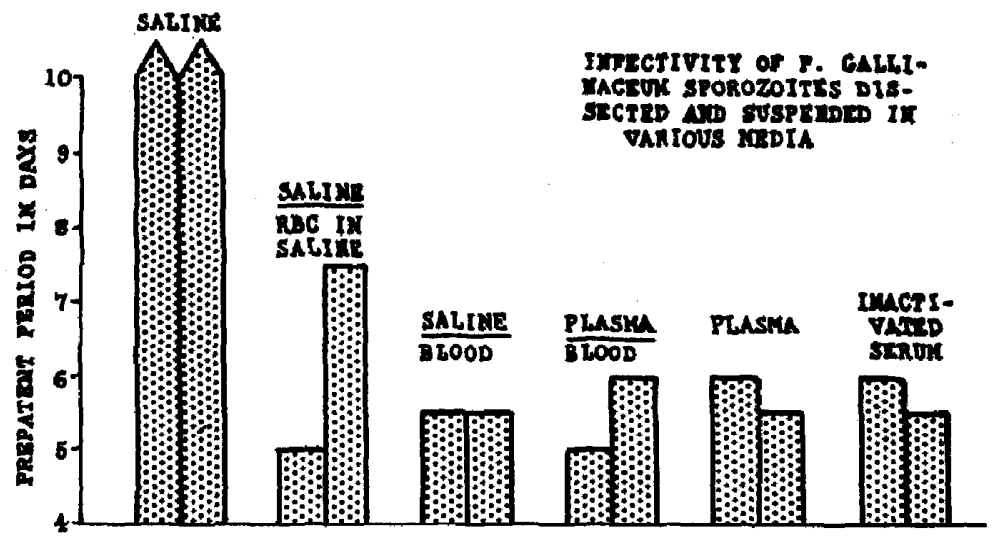

FIg. 6

Figure 6 shows results of a comparison of various dissecting and suspending media. Where one medium is given, as for the first two bars, it was used for both dissection and suspension; where two are given, as in the third and fourth bars, the medium above the line was used for 
dissection and that below the line for suspension. As in the experiments shown in Fig. 5, 0.85\% saline was harmful, no infections resulting. Washed erythrocytes in saline gave erratic results, perhaps because of small amounts of plasma in the medium. Blood, heparinized plasma, and serum inactivated at $56^{\circ} \mathrm{C}$ for 30 minutes were equally satisfactory media.

Figure 7 shows the effects of suspending media consisting of 'saline with varying amounts of blood. The general procedure here was as in

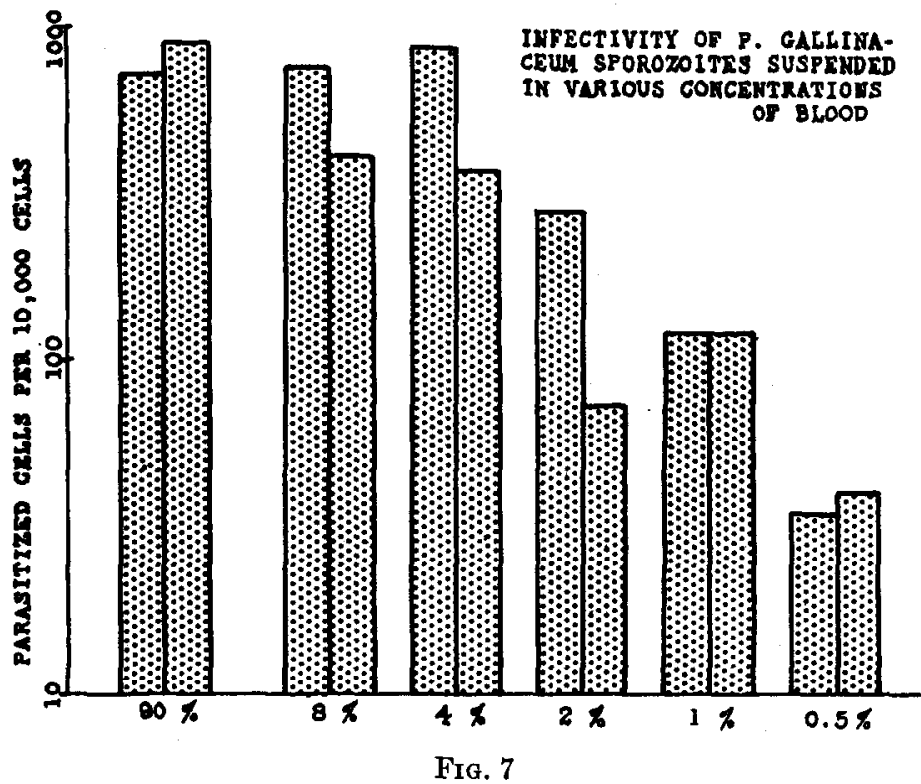

the experiment illustrated by Fig. 6 , but each mosquito sample was dissected in $0.85 \% \mathrm{NaCl}$ solution. The tissue containing the salivary glands was then transferred to $0.85 \%$ saline solution containing the stated percentage of heparinized whole blood. Grinding and immediate inoculation of chickens were carried out in this medium. The parasitemias recorded are thase for the sixth day after inoculation. The adverse effect of concentrations of blood below $4 \%$ is slight but clear.

Figures 8,9 and 10 illustrate data from experiments in which suspensions of sporozoites in $90 \%$ blood were distributed in various media for storage before inoculation. All preparations, therefore, contain a small amount (stated in each case, below) of blood from the original suspension. 
Figure 8 gives results of an experiment comparing survival of sporozoites in various saline solutions (with $4.5 \%$ blood from the original suspension). Storage of the suspensions was for 3-4 hours at about $23^{\circ} \mathrm{C}$. Of the saline solutions studied, $0.85 \%$ was definitely most favorable. In fact, the infections in chicks inoculated with suspensions held for $3-4$ hours in $0.85 \%$ saline with $4.5 \%$ blood were not significantly less intense than those in control chicks inoculated immediately with similar suspensions (arrows at left of graph).

Figure 9 shows the combined effect of medium and temperature in a similar experiment. All media contained $0.5 \%$ blood carried over with

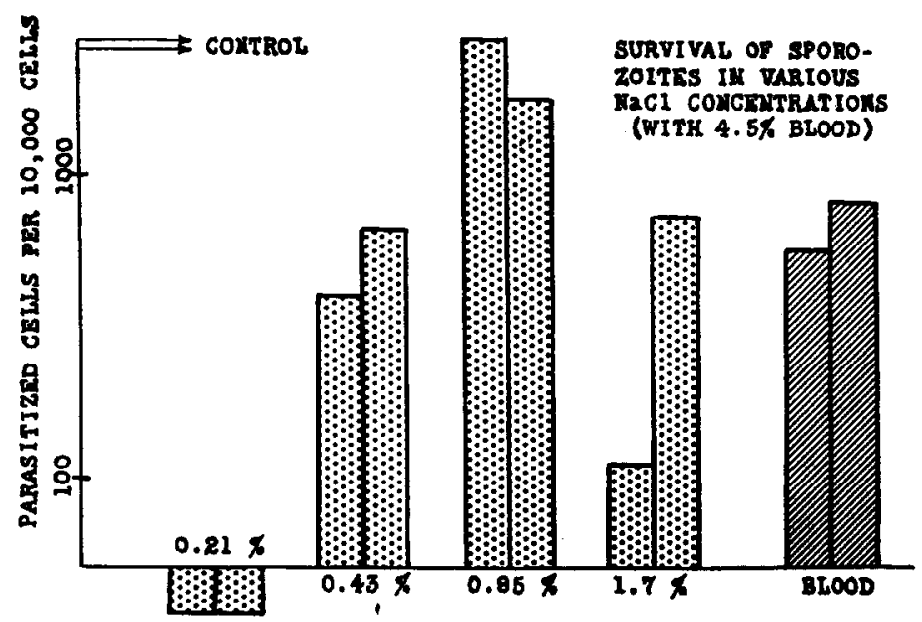

FIG. 8

the sporozoites from the original suspension. Storage was for $3 \frac{3}{4}$ to $4 \frac{1}{4}$ hours at the temperatures given. Arrows at the left show the parasitemias in controls inoculated immediately (without storage) with comparable suspensions in saline-blood. Little loss of infectivity occurred at $16^{\circ} \mathrm{C}$. All media showed considerable loss at $26^{\circ} \mathrm{C}$. At $21^{\circ} \mathrm{C}$ is seen the unfavorable effect of normal serum, as compared with saline-blood or serum inactivated at $56^{\circ} \mathrm{C}$ for 30 minutes.

Figure 10 shows two similar experiments with storage at various temperatures. The data for Experiment II are the data on saline-blood suspensions given in Fig. 9, with $0.5 \%$ blood in $0.85 \%$ saline and storage for $3 \frac{3}{4}$ to $4 \frac{1}{4}$ hours. In Experiment I, the blood concentration was also $0.5 \%$, and the storage period of the suspensions was 3 hours, 55 minutes, to 4 hours, 10 minutes. 


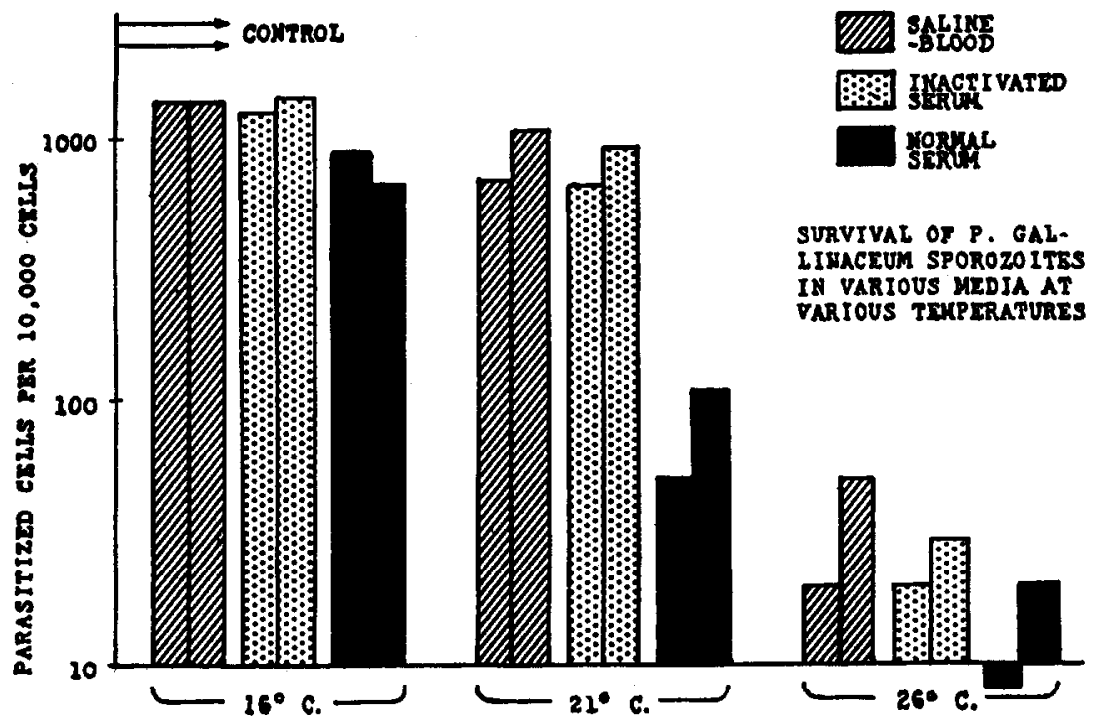

Fra. 9

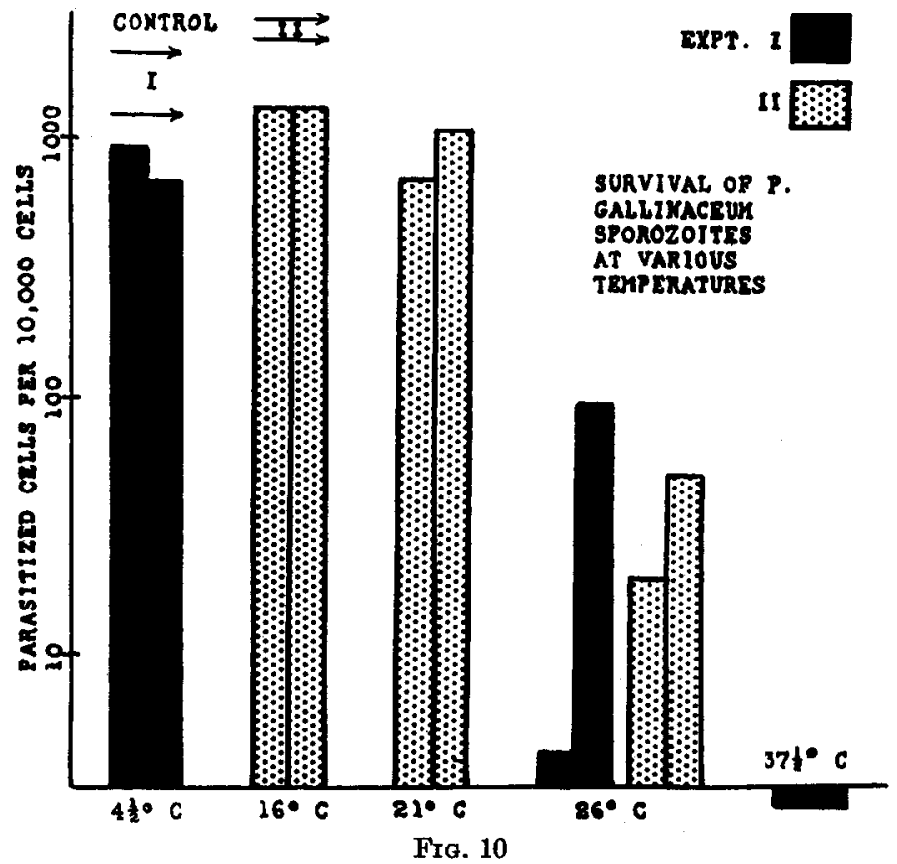


In both experiments the unfavorable effect of temperatures above $21^{\circ} \mathrm{C}$ is clear. It should be noted that this temperature threshold is below the temperatures at which sporozoites survived well in mosquitoes $\left(28^{\circ} \mathrm{C}\right)$ and much below those they encounter in inoculated birds.
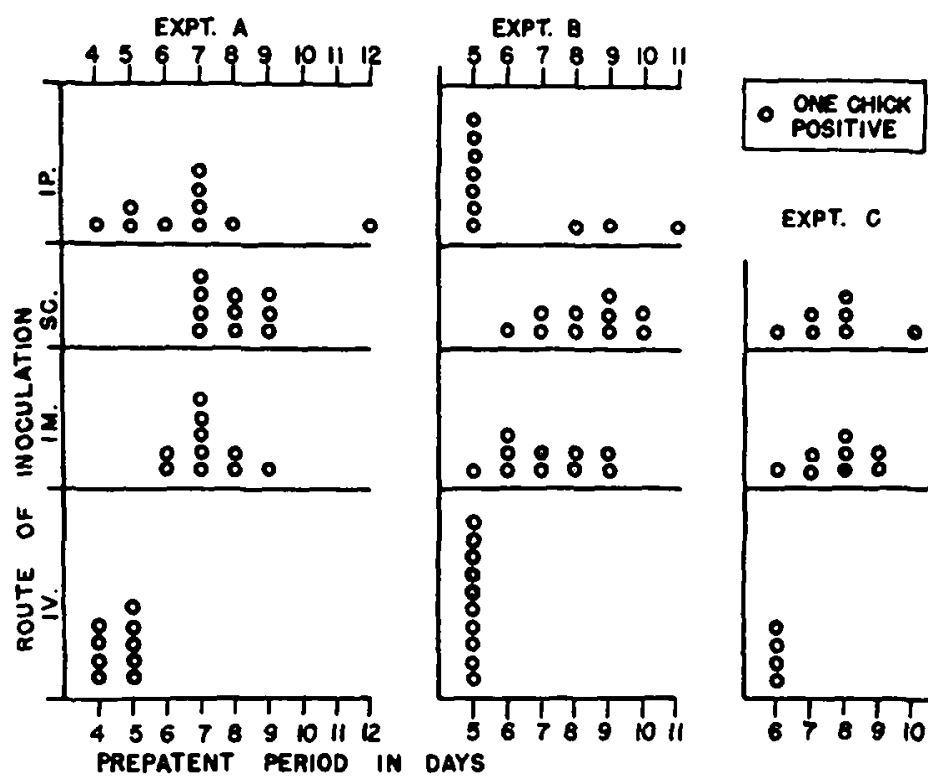

EXPT. C

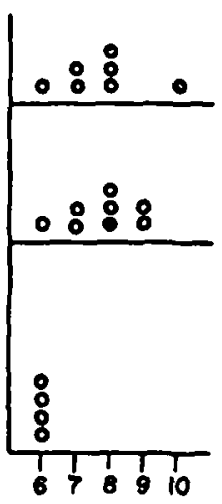

FIa. 11

\section{Route of Inoculation}

Figures 11 and 12 give results from six experiments comparing prepatent periods in chicks inoculated with sporozoites by various routes. Each circle represents one chick which first showed blood parasites on the day indicated after inoculation. The arrow heads in Experiments D and $E$ represent chicks which did not show infection; the duration of observation in these cases is indicated below. The volume of inoculum was $0.2 \mathrm{ml}$ per chicken and the estimated number of sporozoites in each experiment was the same for all groups, except as follows: In Experiment $\mathrm{C}$ each chick received $0.25 \mathrm{ml}$. In Experiment D some of the chicks inoculated intravenously received $0.2 \mathrm{ml}$ each containing 140,000 , while others received $0.2 \mathrm{ml}$ each containing 1,400,000 sporozoites; those inoculated intragastrically (by stomach tube) or orally each received $1 \mathrm{ml}$ con- 
taining 700,000 sporozoites; in Experiment $\mathrm{E}$ the intravenous doses were $0.4 \mathrm{ml}$ containing 60,000 sporozoites (introduced into wing, leg or jugular vein), the intrahepatic and intracerebral doses were $0.03 \mathrm{ml}$ containing 20,000 sporozoites and the intragastric doses were $0.5 \mathrm{ml}$ containing 75,000 sporozoites; in Fxperiment $\mathrm{F}$ both intravenous and intrahepatic doses were $0.03 \mathrm{ml}$ containing 24,000 sporozoites. Thus, only in Experiments $\mathrm{D}$ and $\mathrm{E}$ were the doses of sporozoites for different groups unequal. The inequalities are considered entirely insignificant in relation to the

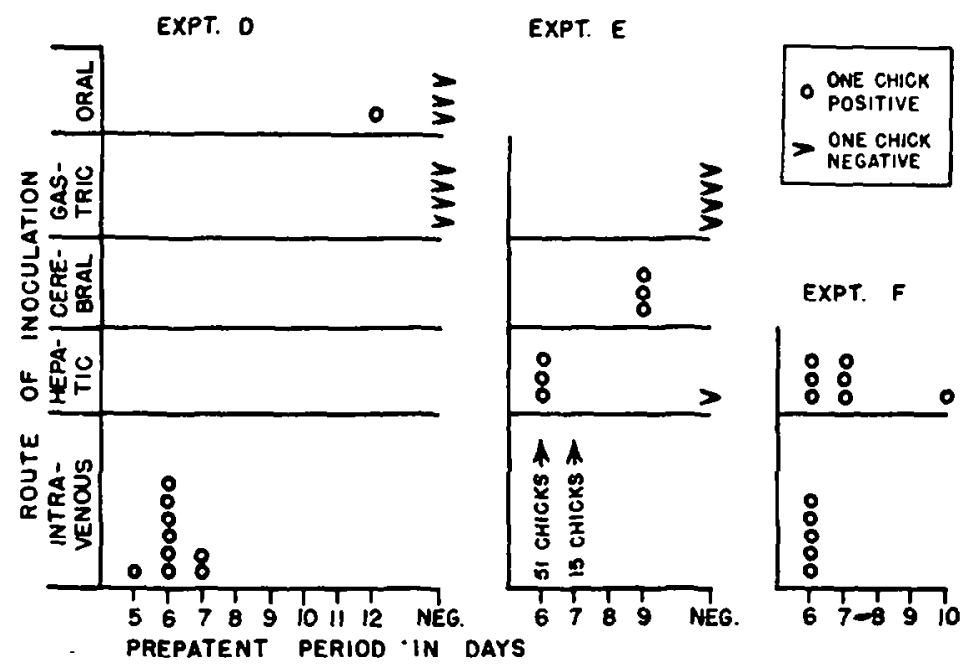

Fig. 12

differences in prepatent periods. The estimated numbers of sporozoites given above are of value for comparison only between groups within each experiment, since factors, such as age, affecting the infectivity of sporozoites were not controlled.

It is clear from Experiments A, B and $\mathrm{C}$ that intravenous inoculation resulted in regular, short prepatent periods. In contrast, intramuscular (breast or thigh), subcutaneous (breast or back) and intraperitoneal inoculation gave less regular and generally longer prepatent periods. It is suspected that the seven intraperitoneally injected chicks with prepatent periods of $\overline{5}$ days in Experiment $\mathbf{B}$ actually received some or all of the dose in the liver, an accident difficult to avoid in intra-abdominal injection of young chicks. This was a major reason for testing direct 
intrahepatic inoculation in Experiments $\mathrm{E}$ and $\mathrm{F}$, where it is apparent that the results agree generally with those of intravenous injection, with occasional notable exceptions. The chick in Experiment $\mathrm{E}$ which did not show parasites was observed for only 11 days, a period insufficient to rule out late infection.

The data on intracerebral inoculation (Experiment E) are insufficient for generalization, but it is clear that this route interfered with rapid development of the infection.

With one significant exception (Experiment D), the oral inoculations and those made by stomach tube gave regularly negative results. The periods of observation in negative birds were 21 days in Experiment D and 33 days in Experiment E. In our experience sporozoite-induced infections have become patent either within 12 days from inoculation or not at all. It is thought, therefore, that observation for 21 days is sufficient to rule out infection.

\section{Discussion}

The experiments summarized above cover all aspects of routine inoculation of sporozoites except the procedures involved in estimating dosage. These last are reserved for another paper (Porter et al., in prep.), since the studies revealed important effects of the age of sporozoites on their infectivity.

It should be noted that determination of prepatent periods or parasitemia at a given time in inoculated chicks does not necessarily measure survival or destruction of sporozoites. Agglutination of sporozoites (Mulligan et al., 1940), for instance, may well reduce the intensity of infection in chicks even though no destruction of sporozoites occurs. Various other possibilities exist, such as adhesion of sporozoites to tissue debris etc., in the suspension. Suffice it to say that the data presented concern the quantitative infections produced and are not necessarily concerned with viability of sporozoites per se.

The studies on methods for immobilizing mosquitoes show that excess ether or temperatures below $\mathrm{O}^{\circ} \mathrm{C}$ drastically reduced the infectivity of sporozoites. That this effect is not merely a result of death of the infected mosquitoes is indicated by the fact that tobacco smoke and pyrethrum did not significantly affect the infectivity of sporozoites when mosquitoes were killed with them.

Previous investigations have revealed the inadequacy of saline solutions for survival of malarial sporozoites in vitro. Porter (1942) reported 
that canary serum or whole heparinized blood were superior to $0.85 \%$ $\mathrm{NaCl}$ solution for survival of sporozoites of $P$. cathemerium. Tonkin (1947) reported poor survival of $P$. gallinaceum sporozoites in saline or Ringer's solution, good survival in heparinized chick plasma or mixtures of as little as $25 \%$ plasma in saline. Whitman (1948) observed that erythrocytic extract obviated the unfavorable action of saline solutions.

The present studies show that, when sporozoites were mechanically separated from the infected glands, saline solutions were uniformly destructive. This was true in salt solutions of $0.85,0.43$ and $0.21 \%$. In the presence of a small amount of blood, saline solutions of $1.7,0.43$, and $0.21 \%$ were less favorable than $0.85 \%$. This is somewhat surprising in view of the low asmotic concentration of the body fluids of insects.

Heparinized blood, heparinized plasma, and inactivated serum were satisfactory media for sporozoites in the present study. The favorable effect of blood on survival of sporozoites was evident in concentrations down to $4 \%$ in saline. Below this concentration, infectivity fell off rapidly. Normal serum showed variable but always unfavorable effects, and it is clear that chicken serum contains a thermolabile substance which reduces the infectivity of sporozoites. Whether this substance is complement or one of its fractions was not determined.

The suggestive data of Haas et al. (1949) on deleterious effects of tissue extracts on sporozoites should be compared here. However, there is no evidence concerning the possible identity of the substance studied by them and the substance present in normal chicken serum. As indicated above, it is possible that effects such as the agglutination observed by Mulligan et al. (1940) in normal serum could reduce infectivity of suspensions of sporozoites.

The effect of elevated temperatures on sporozoites has been reported by Brackett and Hughes (1945), Davey (1946), Whitman (1948), and Tonkin (1947). The present data indicate little effect with temperatures up to $21^{\circ} \mathrm{C}$. Above this point the loss of infectivity was pronounced. It is apparent that the effect here is not direct, but an augmentation of the adverse effect of unfavorable suspending media, since sporozoites survive many days in the salivary glands of infected mosquitoes at temperatures above those shown to be deleterious in vitro.

The data on route of inoculation of sporozoites show marked irregularity of infection with all routes except intravenous and intrahepatic. This suggests either that cryptozoites develop better in macrophages in contact with the blood or that merozoites produced by cryptozoites in such sites are more likely to invade erythrocytes. The nearly uniform 
failure of introductions into the alimentary tract accentuates the single successful oral infection. It seems most likely that this infection resulted from entry of a few sporozoites through an abrasion in the upper alimentary tract.

Concerning the development of a reliable quantitative method for manipulation of sporozoites, the data provide useful information. The following procedure is recommended: Mosquitoes should be immobilized with minimal exposure to ether or with tobacco smoke. The salivary glands should be dissected out in $0.85 \%$ sodium chloride solution and suspended in heparinized normal chicken blood. Aliquots of the suspension prepared in this way should be inoculated intravenously. As we have shown elsewhere (Porter et al., in prep.), if mosquitoes are used within a few days of salivary gland invasion, highly predictable quantitative infections can be produced by inoculation of known numbers of sporozoites with this procedure.

\section{SUMMARY}

Data are presented on the infectivity of sporozoites of $P$. gallinaceum under various conditions. Mosquitoes exposed to ether in excess or to temperatures below freezing showed drastic reduction of infectivity. Considerable exposure to tobacco smoke, DDT or pyrethrum, minimal exposure to ether, or chilling at temperatures above freezing were apparently harmless to sporozoites.

Saline solutions of $0.21,0.43$ and $0.85 \%$ were injurious to sporozoites. In the presence of $4.5 \%$ blood $0.85 \%$ saline was less deleterious than more or less concentrated saline solutions. Heparinized chick blood, plasma, or serum inactivated at $56^{\circ} \mathrm{C}$ for 30 minutes were good media, allowing prolonged survival of sporozoites. Normal serum slightly but definitely reduced infectivity. Temperatures up to $21^{\circ} \mathrm{C}$ allowed nearly quantitative survival of sporozoites for $3-4$ hours. Temperatures of $26^{\circ} \mathrm{C}$ or higher markedly reduced infectivity within this period.

Intravenous inoculation of sporozoites gave regular, intense infections. Intrahepatic injection was apparently nearly as good. Intramuscular, subcutaneous, and intraperitoneal inoculations resulted in irregular infections of mostly prolonged incubation period. Inoculations by stomach tube were negative. One of four orally inoculated chicks became infected with a prolonged prepatent period.

A convenient method is described for routine quantitative induction of infection with sporozoites of Plasmodium gallinaceum. 


\section{REFERENCES}

BracketT, S., and Hughes, C. O. 1945. Chilling as a means of retaining the viability of the sporozoites of Plasmodium gallinaceum. J. Parasitol. 31, 288-289.

Coggeshald, L. T., Porter, R. J., and Laird, R. L. 1944. Prophylactic and curative effects of certain sulfonamide compounds on exoerythrocytic stages in Plasmodium gallinaceum malaria. Proc. Soc. Exptl. Biol. Med. 67, 286-292.

DAver, D. G. 1946. Use of avian malaria for discovery of drugs effective in treatment and prevention of human malaria; drugs for causal prophylaxis and radical cure or chemotherapy of exo-erythrocytic forms. Ann. Trop. Med. Parasitol. 40, 453-471.

Hass, V. H., Wilcox, A., and Coleman, N. 1949. Modification of P. gallinaceum infections by certain tissue extracts. J. Natl. Malaria Soc. 8, 85-99.

Mulligan, H. W., Russeli, P. F., and Mohan, B. N. 1940. Specific agglutination of sporozoites. J. Malaria Inst. India 3, 513-524.

Ponter, R. J. 1942. Tissuc distribution of exoerythrocytic schizonts in sporozoiteinduced infections with Plasmodium cathemerium. J. Infectious Diseases 71, $1-17$.

Porter, R. J., Laird, R. L., and Dusseau, E. M. Studies on malarial sporozoites. II. Relation of age and numbers to infectivity of sporozoites. In prep.

Shannon, R. C., and Putnam, P. 1934. The biology of Stegomyia under laboratory conditions. Proc. Entomol. Soc. Washington 36, 185-242.

TonkIN, I. M. 1947. Influence of suspending fluid on survival of sporozoites in vitro. Trans. Roy. Soc. Trop. Med. Hyg. 41, 259-262.

Whitman, L. 1948. Prolonged viability of sporozoites of Plasmodium gallinaceum in extracts of washed chicken erythrocytes. $J$. Immunol. 69, 285-294. 